\title{
基于微课的翻转课堂教学模式在分析化学实验教学中的探索
}

耿萍, 张帆 ${ }^{*}$

华东师范大学化学与分子工程学院, 上海 200241

摘要: 分析化学实验是大学化学实验的重要内容, 是分析化学课程学习中不可缺少的组成部分。为了提高分析化学实验 教学质量, 提升学生动手能力、分析问题和解决问题的能力以及创新能力, 解决传统教学模式存在的主要问题, 对分析 化学实验教学模式进行了改革探索。将以微课为基础的翻转课堂教学模式引入到分析化学实验中, 激发学生的实验热情, 提高学生综合实验技能，巩固基础理论知识，培养了科学研究思维，真正实现创新性人才培养目标。

关键词: 翻转课堂; 分析化学实验教学; 微课; 教学模式

中图分类号: G64; O6

\section{Exploration of Flipped Classroom Teaching Mode Based on Micro-Course in Analytical Chemistry Laboratory Teaching}

Ping Geng, Fan Zhang *

School of Chemistry and Molecular Engineering, East China Normal University, Shanghai 200241, P. R. China.

\begin{abstract}
Analytical chemistry laboratory is an important part of chemistry laboratory and analytical chemistry learning. In order to improve the teaching quality, practical ability, problem-solving ability and innovative ability of students, the flipped classroom teaching mode based on micro-course has been applied in the analytical chemistry laboratory. It is proved that this teaching mode aroused the learning passion, improved the comprehensive experimental skills, consolidated the theoretical knowledge, and cultivated scientific research thinking, thus, really achieved the goal of creative talents.
\end{abstract}

Key Words: Flipped classroom; Analytical chemistry laboratory teaching; Micro-course; Teaching mode

分析化学实验是化学及相关专业一门重要的基础实验课程, 是分析化学课程教学中不可或缺的 重要组成部分, 更是将分析化学理论知识应用于操作实践的重要平台 ${ }^{[1-3]}$ 。分析化学实验既能培养学 生严谨求实的科学态度、认真细致的科学实验习惯和积极探索的实验精神, 又能锻炼学生观察、分 析、解决问题的能力, 激发学生的思维能力和创新能力。如何有效地完成分析化学实验所肩负的重 任, 一直是创新性人才培养过程中值得深思的问题 ${ }^{4-6]}$ 。在长期的分析化学实验教学过程中, 通过总 结和研究, 根据现今创新人才培养的理念, 结合我校实际对分析化学实验教学进行了积极的改革和 探索, 提出了以微课为基础的翻转课堂教学模式, 让学生在实验过程中切身感受理论与实践的结合, 培养其科学的思维方式，激发他们独立思考、自主学习的积极性及创新意识。

收稿: 2019-04-18; 录用: 2019-05-06; 网络发表: 2019-05-29

“通讯作者, Email: fzhang@chem.ecnu.edu.cn

基金资助：国家自然科学基金面上项目(21575042); 华东师范大学实验技术研究项目 


\section{1 分析化学实验课程教学现状}

\section{1 学生主动性不足}

分析化学实验针对本科二年级的学生开设, 是学生系统接受实验技能训练的开端。由于实验课 时不足, 实验安排与理论教学往往不能同步, 学生在实验课上经常照本宣科, 机械地根据实验讲义 和教师的讲解做实验, 不善动脑, 处于被动学习的状态, 无法真正实现理论与实践的结合, 无法达 到实验教学的根本目的。同时, 实验教学内容也是直接影响学生主动性的重要因素。分析化学实验 以滴定实验居多, 内容枯燥, 重复性高, 无法充分调动学生的积极性与主动性, 不利于培养学生的 创新思维, 提高其创新能力。

\section{2 教学互动性差}

在目前的分析化学实验教学过程中, 教学方式仍然比较单一, 教师通常以 PPT 课件和板书的形 式为主。首先讲解实验原理和实验步骤, 然后由学生机械地模仿完成实验操作, 最后学生上交实验 报告。实验前学生的预习流于形式, 对实验中出现的问题有较差的预见性, 教师也无法获知学生的 预习情况, 教学缺乏针对性。在这一传统教学模式中, 教师依然是教学的中心, 起着主导作用, 学 生只是被动地接受学习, 缺乏积极思考的能力, 影响了学生主观能动性的发挥, 难以激发学生的学 习兴趣。

\section{3 考核机制不完善}

实验报告是目前实验成绩评定的重要依据, 主要侧重于实验报告的书写、实验数据的记录和分 析等, 对实验过程、科学思维等方面的评定较少, 导致了实验成绩评价方式过于单一。有的学生对 实验原理一知半解, 实验过程中操作不认真, 甚至一些基本操作不规范, 而这些在实验报告中都无 法体现。实验考核机制的不完善, 不利于全面考查学生的综合实验素质, 在一定程度上也会降低学 生做实验的热情以及深入思考的积极性。

\section{2 分析化学实验教学中引入微课的意义}

分析化学实验课程重点考查学生的实际动手操作能力, 是培养学生专业技能的重要实践环节, 学生在做实验的过程中也能够进一步巩固和掌握分析化学理论知识。这门课程包含称量、溶液配制、 移液、滴定等多个单元操作, 每一个单元操作中又包含很多操作要点, 而在实验教学中, 教师很难 向每位学生清楚演示实验的基本操作和规范动作, 使得学生很难准确掌握这些实验技能, 进而影响 了实验结果的准确性。微课全称 “微型视频课程” , 运用建构主义方法, 以教学视频为主要授课方 式, 是围绕某一具体的知识点进行的教学过程及相关资源的有机整合 ${ }^{[7-9]}$ 。微课的核心是微视频, 时 长一般在 5-15 分钟, 具有短小精悍的特点。将分析化学实验操作制作成微视频, 可以通过镜头的推 进, 多方位展示操作过程, 大大增强了实验的演示效果, 进而解决目前教学过程中存在的各种问题, 因此将 “微课”引入分析化学实验教学十分必要。

\section{1 丰富教学方式, 优化教学效果}

通过观看微视频, 整个实验流程在学生脑海中可以直观、生动地呈现, 比仅仅预习教材上的内 容更容易理解和记忆, 对实验原理、实验操作能够有更深刻的了解。比如在滴定操作中, 滴定终点 的判断是难点, 特别是在有些实验中, 溶液变色过程有过渡色且颜色接近, 很难直接根据书本描述 判断滴定终点, 导致实验结果准确度降低。通过微视频, 可以直观清楚地对颜色进行辨认, 减小因 终点判断错误带来的实验误差, 学生能够更准确地对实验结果进行分析。这样的微视频教学可以激 发学生的实验热情和学习兴趣, 使学生的学习由被动变主动, 进而提升教学效果, 提高教学质量。

\section{2 满足学生个性化的学习需求}

由于每个学生的理解能力和知识水平有很大的差异性, 因此在实验中衍生的问题自然不同。分 析化学实验中的基本操作, 如电子天平的差减称量、滴定管的气泡排除和正确检漏、移液管和容量 瓶的使用等, 每一步操作所产生的误差都会产生叠加效应传递到下一步, 从而严重影响实验结果。 
将实验相关教学内容制作成微视频, 学生可以有计划地自主选择相关内容进行学习。微视频教学可 以满足学生个性化的学习需求，提高实验课堂的教学效率。

\section{3 反复播放, 加深印象}

在传统的实验教学过程中, 教师讲解是主要的教学方式。如果学生在听课时开小差或是没听清 楚, 导致理解、记忆有偏差, 就会面临实验失败或实验数据不准确等问题。通过微视频, 学生可以 反复观看学习实验教学流程, 充分理解相关知识要点, 掌握操作技能。

\section{3 基于微课的翻转课堂教学模式用于分析化学实验教学}

翻转课堂教学模式最早由美国学者 J. Wesley Baker 于 2000 年正式提出, 自 2011 年开始逐渐 进入全球教育研究界的视野, 成为了各级各界教育工作者的研究热点。翻转课堂颠倒了传统教学 模式的教学顺序, 重组了传统课堂的教学结构和流程。将先教师课内讲授, 后学生课外进行知识 内化, 颠倒为先学生课外自主学习, 后课内在教师的指导下通过交流、讨论、实验等方式达到知识 内化 ${ }^{[10,11]}$ 。近年来, 国内教育工作者就翻转课堂教学模式进行了大量的研究, 并将其成功地应用于 课程教学中 ${ }^{[12,13]}$ 。以翻转课堂教学模式理念为基础并结合本学院分析化学实验教学的特点, 我们对 基于微课的翻转课堂教学模式进行了探索和实践, 该教学模式主要由课前学习、课堂活动和课后评 价与反馈三个部分组成, 其流程如图 1 所示。

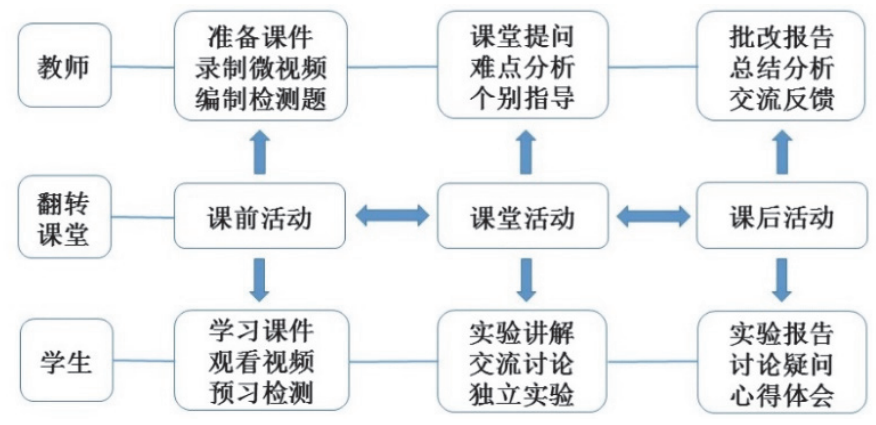

图 1 翻转课堂教学流程图

\section{1 课前学习}

课前自主学习是翻转课堂教学模式有效实施的关键, 学生自主学习的质量直接关系到后续课堂 活动的顺利进行。因此, 为保证学生的课前自主学习质量, 针对每个实验, 教师设计和制作带有讲 解音频的教学课件 (包括实验目的、实验原理、实验步骤等), 玻璃器具和仪器设备的使用和操作过程 微视频以及实验过程中重要操作步骤的微视频, 并将这些教学资源上传至学校教务处建设的 “大夏 学堂” 教学平台。学生登录 “大夏学堂”, 能够方便地按照自己的进度需求进行自主学习, 完成预 习报告的书写, 并设置了课前预习测试题, 完成预习作业的学生才可以开展实验。为了督促学生完 成课前学习, 我们对教学平台进行了设置, 比如首次观看实验微视频不能拖动播放器的进度条, 也 不能最小化, 必须观看完成后才能进行其他操作, 这样可以保证课前学习的效果。同时, 如果学生 在自主学习过程中遇到问题, 也可通过 “大夏学堂” 平台上的讨论区反馈给教师。教师可以对学生 的预习情况进行追踪和查看, 并对学生反馈的问题进行整理、汇总, 及时调整教学方法和授课内容, 有针对性地组织后续课堂活动。

\section{2 课堂活动}

课堂知识内化阶段是翻转课堂教学模式的核心, 是通过课堂活动完成的。因此, 设计有效的课 堂活动对促进学生完成对知识的内化至关重要。传统的实验教学模式是 “教师讲、学生听”, 这种 教学方式不利于学生动手能力的培养, 学生积极性不高, 教学效果差。为了提高分析化学实验教学 与师范院校教育特色的契合度, 我们在课堂中做了一些教学尝试, 进行角色转换, 以教促学, 提高 
学生的综合能力。学生通过 “大夏学堂” 教学平台上的微视频和实验讲义完成预习, 课堂上轮流讲 解实验原理、实验内容和实验操作。教师通过课堂集中提问和交流讨论检查学生的自学情况, 以督 促学生在课前完成高质量的自学; 根据学生的自学情况及反馈的问题, 对一些共性问题进行集中讨 论和答疑; 对重要的知识点进行梳理和强调, 并设计问题引导学生在实验过程中进行思考。在课堂 实施过程中, 教师的讲解时间大大缩短, 主要时间用于给学生进行实验操作、问题思考及讨论交流。 通过汇总实验中的问题, 形成新的教学资源, 为后续开展的翻转课堂学习提供素材。由于完成了课 前学习, 学生实验操作的规范性和准确性得到了进一步的提高和保障。通过学生自主学习和教师针 对性的讲解, 实现了对知识的吸收和内化。

\section{3 课后评价与反馈}

课后评价与反馈是翻转课堂教学模式的延伸, 教师对学生提交的实验报告进行批改, 把实验报 告中存在的问题及时通过 “大夏学堂” 教学平台与学生进行沟通和讨论, 对各类问题进行归纳, 并 引导学生完成知识的总结和提升。同时, 建立客观反映学生综合实验素质的考核方法非常必要。分 析化学实验成绩的评价采用多元化的方式, 包括自主学习情况、实验过程、实验结果等多个方面的 考核, 学生的讲课表现也是实验成绩考核标准之一, 这样可以充分调动学生的主观能动性。在分析 化学实验的总成绩中, 包括课前预习 $15 \%$, 课堂表现 $30 \%$, 实验报告 $25 \%$ 和考核实验成绩 $30 \%$ 。这 样可以全面客观地反映出学生的学习效果, 调动了学生的积极性, 提高了学生的实验兴趣和实验能 力。

\section{4 实践过程实例分析}

我们以 “EDTA 溶液的配制与标定” 实验为例, 探讨基于微课的翻转课堂教学模式在实验教学 中的应用。

\subsection{1 课前活动}

准备教学课件: 设计和制作带有讲解音频的教学课件(包括实验目的、实验原理、实验步骤, 实 验数据记录), 上传至 “大夏学堂” 网站供学生预习观看。

录制实验微视频：本实验中涉及的分析化学实验基本操作较多，我们将 “酸式滴定管的使用” “电子天平的使用” “称量瓶的使用” “移液管的使用” “容量瓶的使用” 和 “实验终点的确定” 这些操作过程及注意事项录制成微视频, 放在 “大夏学堂”网站上供学生反复观看学习。

编制预习检测题：根据本次实验课的教学目的编制了以下预习测试题：

测试题 1：标定 EDTA 浓度的常用工作基准物质有哪些？应如何选择？

测试题 2: 在配位滴定中为什么要加入缓冲溶液?

测试题 3: 用钙标准溶液标定 EDTA 溶液时, 为什么要先制备 EDTA-Mg 配合物溶液, 然后再 加入钙标准溶液, 从而标定 EDTA 溶液?

学生利用课余时间观看课件和视频, 完成预习检测, 并可将疑问反馈到 “大夏学堂” 交流区, 教师对学生的预习情况进行追踪和查看, 并对学生反馈的问题进行整理、汇总, 有针对性地设计和 调整后续课堂活动内容。

\subsection{2 课堂活动}

通过课件和微视频的学习, 学生已对实验内容有了基本了解, 课堂上教师针对学生的疑惑进行 讲解, 并对以下问题进行思考讨论:

问题 1: 配制 EDTA 溶液时, 为什么先在烧杯中溶解后转移到试剂瓶中, 能否直接在试剂瓶中 溶解?

问题 2: 为什么要在 $\mathrm{pH}=10$ 的缓冲溶液中使用铬黑 $\mathrm{T}$ ?

问题 3: 为什么用乙二胺四乙酸的二钠盐配制 EDTA 溶液，而不用其酸?

问题 4: 怎样掌握好终点?

讨论时间为 40 分钟, 然后学生开始独立完成实验。实验过程中教师跟踪指导, 及时指出实验中 的错误操作, 实验操作时间为 100-120 分钟。实验操作结束后, 学生当场完成实验报告, 教师检查 
学生实验数据记录和实验结果的合理性与科学性, 签字确认。

\subsection{3 课后活动}

学生提交实验过程的总结和反思, 尤其对实验的得失成败做一个小结。教师对学生的实验报告 及总结反思进行评阅, 并把实验报告中存在的问题及时通过 “大夏学堂” 教学平台反馈给学生, 对 共性问题进行归纳, 引导学生思考未来实验需要进一步探讨和研究的问题。

\section{4 实践效果}

翻转课堂教学模式树立 “以学生为主体, 教师为主导” 的教学理念, 积极开展启发式、探索式 教学方法, 充分调动学生学习的主动性和创造性, 培养学生的实际应用能力和责任意识。将翻转课 堂教学模式引入分析化学实验中, 学生按照自己的实际水平安排学习进度, 促进了学生对知识的理 解和深化, 有效激发了学生的学习兴趣, 提高了学生自主学习的能力和主观能动性。学生都很珍惜 每一次的讲课机会, 课前准备很充分, 课堂气氛活跃, 学生的踊跃参与, 增强了课堂教学的互动性, 激活了学生的思维。教师只需对实验相关内容进行补充和提问, 有更充裕的时间来解释实验的疑点 和难点, 这是把知识内化的过程放在了课堂上。这种 “先学后教” 的模式颠覆了传统课堂的 “先教 后学” 的模式, 并且在分析化学实验课程中, 更加强调了学生的 “学”, 真正实现 “以学生为中心” 的教学理念。同时, 在 “大夏学堂” 教学平台上, 我们还设立了讨论交流区, 学生可以将实验中遇 到的疑难点和经验教训等发布在交流区, 便于其他同学学习和借鉴, 也促进了师生之间的交流与讨 论。这种翻转课堂教学模式调动了学生学习的能动性, 提高了学习效率和深度, 有助于真正实现以 学生为中心的因材施教和自主学习, 更提高了实验教学质量, 对分析化学实验教学改革起到了积极 的推动作用。

\section{5 结语}

通过实施 “基于微课的分析化学实验翻转课堂教学模式”, 调动了学生的学习积极性和主动性, 促进了学生良好实验习惯的养成, 真正实现了 “学生为主体, 教师为主导” 的宗旨。翻转课堂教学 模式在强调学生技能培养的同时, 提升了学生在学习中探究性学习的能力, 进一步培养了学生的创 新能力, 为创新性人才的培养进行了有益的探索和实践。教学实践证明, 以我校 “大夏学堂” 为平 台, 建设基于微课的翻转课堂分析化学实验教学模式, 是对传统实验课堂的优化和创新, 对于提高 学生的科学动手能力、创新能力、综合设计能力以及团队协作能力有着非常重要的现实意义。

\section{参 考 文 献}

[1] 蔡鹰，李思东，杨否，黄娜，吴湛霞. 实验技术与管理, 2018, 35 (3), 19.

[2] 郭昉, 段佳勇. 实验室研究与探索, 2012, 31 (10), 138.

[3] 韦寿莲, 刘永, 严子军. 化学教育, 2016, 37 (8), 21.

[4] 何英, 龚慧芳. 大学化学, 2013, 28 (3), 24.

[5] 任春艳, 马传利, 吕海涛. 化学教育, 2015, 36 (22), 42.

[6] 陶然, 赵文秀, 董树国, 白羽. 大学化学, 2018, 33 (6), 60 .

[7] 李健军, 许昭, 白艳红, 杨云. 实验室研究与探索, 2017, 36 (1), 189.

[8] 胡铁生, 周晓清. 现代教育技术, 2014, 24 (2), 5 .

[9] 宿艳, 张艳娟, 吴硕, 崔栐, 孟长功. 实验室科学, 2017, 20 (4), 70.

[10] 丁雪梅, 张晓君, 王鹏, 赵云, 李玉梅, 谭智敏, 丛彦龙, 张乃生, 成军. 实验室研究与探索, 2015, 34 (6), 207.

[11] 王红，曾秀琼，刘秋平，梁秋霞，张根荣. 实验技术与管理, 2015, 32 (5), 196.

[12] 冯建，遇丽，熊伟，苏小东，实验技术与管理, 2018, 35 (4), 208.

[13] 王彦沙, 刘松艳. 大学化学, 2016, 31 (4), 17. 